\title{
Caracterização do Comportamento de E/S Utilizando Aprendizado não Supervisionado *
}

\author{
Pablo J. Pavan ${ }^{1}$, Jean Luca Bez ${ }^{1}$, Matheus S. Serpa ${ }^{1}$, \\ Francieli Zanon Boito ${ }^{2}$, Philippe O. A. Navaux ${ }^{1}$ \\ ${ }^{1}$ Universidade Federal do Rio Grande do Sul (UFRGS) - Porto Alegre - RS - Brasil \\ \{pjpavan, jean.bez, msserpa, navaux\}@inf.ufrgs.br \\ ${ }^{2}$ Univ. Grenoble Alpes, Inria, CNRS, Grenoble INP, LIG, 38000 Grenoble, France \\ francieli.zanon-boitodinria.fr

\begin{abstract}
Resumo. Nas aplicações de HPC operações de E/S são gargalos devido a diferença entre velocidade de processamento e de acesso a um dado. Assim, caracterizar as operações pode auxiliar na busca de desempenho. Deste modo, este trabalho propõe uma abordagem com aprendizado não supervisionado para a caracterização de E/S. Utilizando dados do supercomputador Intrepid, fomos capazes de identificar a principal característica de suas aplicações.
\end{abstract}

\section{Introdução}

As operações de E/S são um gargalo de desempenho em grande parte das aplicações devido a diferença entre velocidade de processamento e de acesso de um determinado dado. As operações são caracterizadas por padrões de acessos. Os padrões representam o modo que as aplicações acessam seus dados e este modo reflete no desempenho das mesmas. Portanto, é importante identificar e caracterizar os padrões, dado que assim podemos identificar possíveis gargalos. Com essa premissa, propomos uma abordagem de caracterização de E/S que usa aprendizado não supervisionado empregando o algorítimo $K$-means para agrupar jobs com comportamento de E/S similar usando informações de profiling reportadas pela ferramenta Darshan ${ }^{1}$. Como estudo de caso, aplicamos nossa abordagem em quatro meses (Abril, Maio, Junho e Julho) de atividade - um total de 28.938 jobs — do supercomputador Intrepid localizado no Laboratório Argonne.

\section{Trabalhos relacionados e Metodologia}

Inúmeros trabalhos caracterizam E/S como os de [Zoll et al. 2010,Liu et al. 2016], porém, diferentemente destes, neste trabalho propomos uma abordagem de caracterização, não somente uma análise dos dados. Outros trabalhos como [Di et al. 2014, Calzarossa et al. 2016] aplicam abordagens de aprendizado não supervisionado na caracterização de jobs similares, porém não abordam a E/S considerando os padrões de acessos.

Nossa abordagem parte desde o arquivo gerado pelo Darshan até a utilização do algoritmo K-means. Primeiramente nós extraímos dos arquivos originais fases de E/S, estas representam momentos em que um job realizou uma operação de E/S caracterizada por um padrão de acesso. Após a extração criamos um conjunto de dados interpretado

\footnotetext{
*Trabalho financiado pela Petrobras sobre o projeto 2016/00133-9.

${ }^{1}$ https://www.mcs.anl.gov/research/projects/darshan/
} 
pelo K-means e aplicamos os métodos de Silhouette e Elbow para encontrar o número ótimo de grupos para cada mês. Aplicamos esta abordagem nos meses de forma separada, para podermos identificar as características que se mantiveram através do tempo e as que eram únicas. Para caracterizar cada grupo, foi escolhida a fase com mais porcentagem de tempo de E/S nos jobs, portanto cada grupo possui uma fase com principal característica. Para cada fase principal foi designado uma letra, para melhor compreensão.

\section{Resultados Preliminares e Conclusão}

Para cada mês tivemos números de grupos diferentes, foram 11, 10, 7 e 5 respectivamente para Abril, Maio, Junho e Julho. A Figura 1 mostra a porcentagem que cada fase representa no tempo de E/S de cada mês. O grupo A e o grupo $\mathbf{F}$ ocorrem em todos os meses, sendo que o grupo A apresenta um valor representativo no tempo de E/S de 59\%, 26\%, $46 \%$ e $24 \%$ respectivamente em cada mês. A fase principal deste é caracterizada por acessos de escrita consecutiva em arquivos únicos com tamanho de requisição entre 0 à 100 Bytes utilizando a interface POSIX. Este grupo também apresenta o maior número de jobs $(22,946$ de um total de 28,938$)$, sendo que estes em média só realizam operações durante $2.41 \%$ do seu tempo de execução. Podemos assim concluir que o grupo A é caracterizado por um número alto de jobs que sozinhos realizam poucas operações de $\mathrm{E} / \mathrm{S}$, majoritariamente de escrita, mas juntos são responsáveis por boa parte da carga de E/S nos meses. Outros grupos também apresentam uma porcentagem na carga de trabalho de E/S, porém acontecem em menor frequência, ou somente uma vez. Alguns deles como H, I, M e $\mathbf{O}$ são caracterizados por jobs de uma única aplicação, assim, apresentam diferentes padrões de acessos em uma única fase de $\mathrm{E} / \mathrm{S}$.

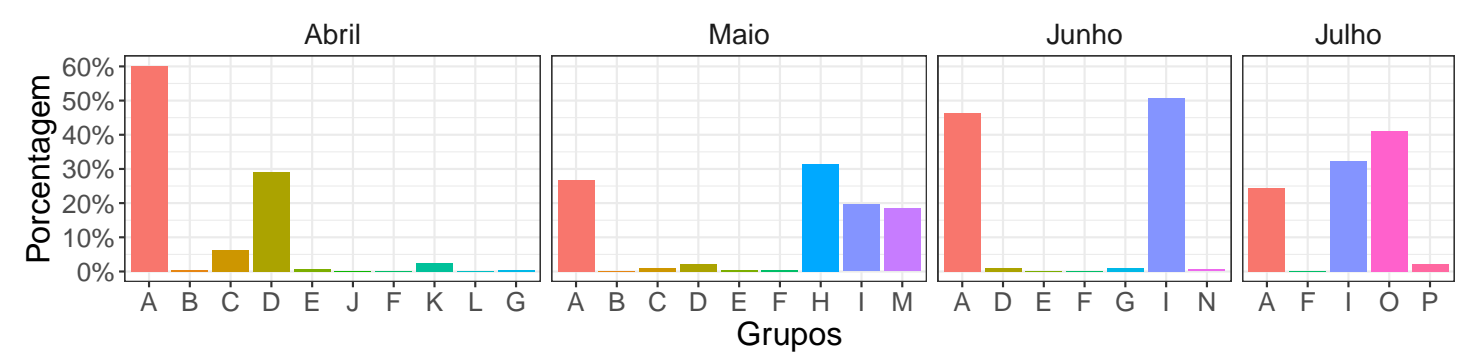

Figura 1. Porcentagem relativa das fases no tempo de E/S de cada mês.

\section{Referências}

Calzarossa, M. C., Massari, L., and Tessera, D. (2016). Workload characterization: A survey revisited. ACM Computing Surveys (CSUR), 48(3):48.

Di, S., Kondo, D., and Cappello, F. (2014). Characterizing and modeling cloud applications/jobs on a google data center. The Journal of Supercomputing, 69(1):139-160.

Liu, Y., Gunasekaran, R., Ma, X., and Vazhkudai, S. S. (2016). Server-side log data analytics for $\mathrm{i} / \mathrm{o}$ workload characterization and coordination on large shared storage systems. In SC'16: International Conference for High Performance Computing, pages 819-829. IEEE.

Zoll, Q., Zhu, Y., and Feng, D. (2010). A study of self-similarity in parallel I/O workloads. In Mass Storage Systems and Technologies (MSST), 2010 IEEE 26th Symposium on, pages 1-6. IEEE. 